\title{
A Clinical Research on the 60 Cases of Intrauterine Asphyxia Rescue
}

\section{and Nursing}

\author{
Yanhua Guan ${ }^{1, a} \quad$ Daoxin Jin ${ }^{1, b}$ \\ ${ }^{1}$ Luohe Medical College, Luohe City, Henan Province, China, 462000 \\ a email: \\ b email:
}

Keywords: Neonatal Asphyxia, First Aid Nursing, Recovery Rate, Complication

\begin{abstract}
Purpose: To explore the effective methods and clinical effect of neonatal asphyxia rescue. Method: To select 60 cases of newborn asphyxia diagnosed and treated in our hospital from January 2014 to 2016 May. After admission, all the children were performed 1 min Apgar score, and immediately taking some measures, such as warm keeping, airway cleaning, breath building and hypoxia correcting. Results: After the formal recovery of our hospital the neonatal asphyxia all achieved success, no complications, no death, and 10 points Apgar score can reach more than 8 points, which has statistical significance $(\mathrm{P}<0.05)$ compared with before recovery. Conclusion: Taking effective first aid and nursing measures can improve the recovery rate and reduce the mortality rate of the newborn, so the clinical effect is remarkable, and the application value is high.
\end{abstract}

\section{Introduction}

Asphyxia of newborn means there is no breathing and only heartbeat after the baby is delivered within 1 minutes. Scoring criteria are based on the skin color, heart rate, muscle tone, respiration and throat reflex. Neonatal asphyxia is an important cause of neonatal death in obstetrics, if the recovery is not in time, the cerebral hypoxia may lead to the development of neonatal nervous system abnormalities. Cesarean section is often used in high risk pregnancy, such as advanced maternal age, pregnancy induced hypertension syndrome, fetal fetal distress, fetal distress, fetal distress and fetal distress. There were more risk factors of neonatal asphyxia in cesarean section, therefore, it is better to do a good job in preparation for resuscitation of asphyxia, in order to be able to carry out the first aid in time, reduce the death rate of neonatal asphyxia, and reduce the incidence of neonatal asphyxia. In order to explore the effective method and clinical effect of neonatal asphyxia rescue nursing. This paper selected 60 cases of neonatal asphyxia from January 2014 to 2016 May, reporting as follows.

\section{Clinical Data}

Selecting 60 cases of neonatal asphyxia in the hospital from January 2014 to May 2016, neonatal birth in 34 cases, cesarean section in 20 cases, vaginal operation in 4 cases and brachial birth in 2 cases.After birth, the Apgar score was performed in all neonates, mild asphyxia neonates (4-7 points) in 49 cases, severe asphyxia neonates (0-3 points) in 11 cases. The clinical diagnosis test got the consent of the patients and their families, and there is no significant difference in general data 
between the patients.

\section{Method}

Prevention of neonatal asphyxia. During childbirth, the nursing staff should strictly observe the birth process, pay attention to maternal uterine contraction, observe and deal with the complications of pregnancy. Taking fetal heart monitoring for the all the production process, if it occurs fetal asphyxia, it must take immediate action to do the rescue measures, such as the change of left lateral position, the maternal oxygen, sodium bicarbonate intravenous rapid input, vitamin $\mathrm{C}$ infusion and accelerating the delivery.

First aid of neonatal asphyxia. After delivery, based on the recovery of neonatal respiratory cycle, it should immediately take on resuscitation of neonatal asphyxia, rapidly remove of neonatal respiratory secretions to ensure airway patency. At the same time, clean the whole body of the newborn and take far infrared radiation to keep warm to avoid neonatal hypothermia. After first aid to establish a self breathing, if the newborn whose heart rate exceeds 100 times / min, it needs to give 5L/min flow pressure oxygen; If the newborn whose heart rate is below $100 / \mathrm{min}$, it needs to immediately carry out positive pressure breathing, every time 30s, continuing to the establishment of independent breathing stop. Most asphyxia neonates are able to recover in a short time, after the airway mucus clearance, mask oxygen or positive pressure ventilation and other first aid. Some severe asphyxia neonates need immediate medication if there is still not reaction after positive pressure ventilation. Pay attention to indications selection and dosage control, and observe the neonatal resuscitation and adverse reactions.

The Nursing of Newborn after the Resuscitation of Asphyxia. After the Neonatal resuscitation, it should take nursing measures, such as warmth retention, maintaining airway patency, Oxygen inhalation, vital signs monitoring, nutrition support and infection prevention. Warmth retention: Due to asphyxia, the newborn delivery abnormal body temperature will drop to trigger a series of adverse symptoms, which will bring many adverse effects on treatment. Therefore, after taking a warm towel to clean the body surface, it needs to use hot water bag or far infrared radiation to keep warm, keeping the newborn body temperature at 36.5 degrees Celsius. Maintaining airway patency: Nursing staff put the newborn to the side of the head lying position, clear airway secretions in a timely manner and maintain airway atency. The operation should be gentle to avoid to stimulate the respiratory tract of the newborn, causing adverse complications. Oxygen inhalation: According to the clinical symptoms of the newborn to determine the mode of oxygen supply and oxygen flow, if the symptoms of neonatal asphyxia is controlled, the oxygen flow rate should be decreased. Continuous oxygen inhalation of the newborn should be pre warm and wet to prevent dry tracheal mucosa. Vital signs monitoring: Monitoring of vital signs in neonates with 24h, including breathing, body temperature, heart rate, skin color, size and so on, if abnormal, it is required to notify the doctor in time and take appropriate measures. Nutrition support: Poor neonatal recovery can be properly delayed open milk time, pay attention to avoid vomiting caused by suffocation; The baby whose recovery is in good condition can normal early suck. Infection prevention: First aid and nursing operation strictly follow the principle of aseptic operation, rationally use antibiotics for infection prevention. Pay attention to the daily use of physiological saline on the newborn to carry out oral cleaning, do a good job in skin disinfection and nursing, daily replace the umbilical cord, and carry out the umbilical disinfection to keep the skin dry. 
Using SPSS 18.0 software to deal with the date and conducting a $x^{2}$ test, using $\mathrm{n}$ (\%) representation. The measurement data is conducted a t test, using $\mathrm{n}(\bar{X} \pm s)$ representation. $\mathrm{P}<0.05$, the difference was statistically significant.

\section{Result}

After the formal recovery in our hospital neonatal asphyxia cases achieved a success, no complications, no death. Apgar score can reach 8 points or more, so it has a statistical significance $(\mathrm{P}<0.05)$ compared with the cases before recovery, see Table 1

Table 1 Apgar Score Comparison

\begin{tabular}{ccccc}
\hline Point of time & & $0-3$ & $4-7$ & $8-10$ \\
\hline \multirow{2}{*}{ before recovery } & 1 minute & $4(6.67)$ & $56(93.33)$ & $0(0.00)$ \\
after recovery & 5 minutes & $1(1.67)$ & $30(50.0)$ & $29(48.33)$ \\
$x^{2}$ & 10 minutes & $0(0.00)$ & $0(0.00)$ & $60(100.0)$ \\
$P$ & $/$ & 5.93 & 6.21 & 7.47 \\
\hline
\end{tabular}

\section{Discussion}

Neonatal asphyxia is common in clinic, and it has become one of the main causes of neonatal death. At present, Improving the quality of life of newborn, preventing the neonatal asphyxia, taking new obstetric technique, strengthening the monitoring of fetal heart during pregnancy, taking effective first aid measures to improve the recovery rate of newborn and nursing care of newborn infants with asphyxia have become the main principle of first aid and nursing of neonatal asphyxia. Simultaneously, in the process of neonatal rescue, it should strengthen the pregnancy test, prenatal care and cesarean section before the preparation. At the time of the operation, it is important to reduce the occurrence of neonatal asphyxia by the strong monitoring, timely detection and timely measures. In order to improve the survival rate and quality of life, it is supposed to do well in the prevention of prenatal care. The main factors that lead to neonatal asphyxia in cesarean section were derived from maternal and the high risk pregnancy is the main factor. Therefore, doing a good job in prenatal care is an important means to reduce the occurrence of neonatal asphyxia. In addition to these risk factors, there are still unknown causes of neonatal asphyxia. Doing a good job in the preparation of the work, strengthening monitoring during operation and taking effective recovery measures can reduce the mortality and complications of neonatal asphyxia.

In summary, taking effective first aid and nursing measures can improve the neonatal resuscitation rate and reduce neonatal mortality rate, and the clinical effect is remarkable, and the application value is relatively high.

\section{References}


[1] Zhu Xiumei. Nursing effect of clinical pathway on neonatal asphyxia during cesarean section [J]. International Journal of nursing, 2015,1 (18):2515-2516.

[2] Xu Xiaoyan. Operation room rescue and nursing of newborn asphyxia in cesarean section [J]. Chinese Journal of nursing, 2014,20 (7):793-795.

[3] Yang Jing, Tan Ling, Wei Daqiong. Application of neonatal resuscitation pillow in neonatal asphyxia [J]. Journal of nursing management, 2014,14 (2):126-126.

[4] Chen Yanwen. Effect analysis of JCI application in obstetric department to reduce neonatal asphyxia [J]. China maternal and child health care, 2013,28 (11):1709-1711.

[5] Wang Haili, Wang Haisha, Duan Yujuan. Impact on the delivery quality of midwives escort throughout the observation [J]. Journal of reproductive medicine, 2015,24 (5): 415-418. 